\title{
Resistencia mecánica de ladrillos preparados con mezclas de arcilla y lodos provenientes del tratamiento de aguas residuales
}

\section{Mechanical Resistance of bricks made of mixtures of clay and sludge from sewage treatment}

\author{
César Augusto García Ubaque \\ Ingeniero Civil, doctor en Ingeniería. Docente Asistente de la Universidad Dis- \\ trital Francisco José de Caldas. Bogotá, Colombia. \\ Contacto:cagarciau@udistrital.edu.co.

\section{María Camila García Vaca} \\ Ingeniera Química. Estudiante de maestría en Ingeniería Química de la Universi- \\ dad de los Andes. Bogotá, Colombia. Contacto: mc.garcia149@uniandes.edu.co.

\section{Martha Lucía Vaca Bohórquez} \\ Psicóloga, MBA. Consultora e investigadora de la Universidad de los Andes. \\ Bogotá, Colombia. Contacto: ml.vaca68@uniandes.edu.co.
}

Palabras clave: eliminación de residuos, ensayo de materiales, productos de arcilla, residuos industriales.

Key words: clay products, testing of materials, industrial waste disposal.

\section{RESUMEN}

En este artículo se analizan las propiedades mecánicas de resistencia a la compresión y absorción de humedad de ladrillos fabricados con mezclas de arcilla y lodos provenientes de plantas de tratamiento de aguas residuales. Se elaboraron mezclas en proporciones 100:0, 99:1, 95:5, 90:10, 80:20 y 60:40 de arcilla y lodo respectivamente. Los ladrillos fueron calcinados entre $35^{\circ} \mathrm{C}$ y 1100
${ }^{\circ} \mathrm{C}$. Los lodos utilizados provienen de la planta de tratamiento de aguas residuales industriales de la empresa G.M. Colmotores. Se realizaron pruebas de absorción de agua y resistencia mecánica a la compresión. De acuerdo con los resultados obtenidos, se concluyó que el rango o el porcentaje de lodo más adecuado para hacer la mezcla con la arcilla, se encuentra entre el 5 y el $10 \%$, ya que con esta medida, el producto cumple con los estándares de calidad exigidos en Colombia. 


\section{investigación}

\section{ABSTRACT}

This work discusses the mechanical properties of bricks that were made with clay and sludge mixtures. We analyzed 100:0, 99:1, 95:5, 90:10, $80: 20$ and $60: 40$ proportions of clay and sludge. Bricks were cooked between $35^{\circ} \mathrm{C}$ to $1100{ }^{\circ} \mathrm{C}$. The sludge used in this study came from sewage treatment plant of G.M. Colmotors Company. These tests were carried out: Water Absorption and mechanical resistance of bricks compression. The results of this study showed that the best percentage of sludge to mix with clay was $5 \%$ to $10 \%$. Since, this percentage range meets the quality standards of the product demanded in Colombia.

\section{INTRODUCCIÓN}

En la actualidad se viene estudiando el uso de nuevos materiales para la construcción, dentro de los cuales se encuentra el reemplazo de parte de la arcilla utilizada en la producción de ladrillos por otros materiales menos densos. Uno de estos materiales es el lodo proveniente de plantas de tratamiento de aguas residuales PTAR, que se convierte además en una solución de tipo ambiental para este tipo de residuos, pues la generación, tratamiento y disposición final de estos lodos, constituyen una de las limitantes y problemas a resolver en el tratamiento de aguas residuales. Cabe destacar que, en la mayoría de países en vía de desarrollo, no se cuenta con mecanismos acordes a las necesidades ambientales que sean económicamente viables y seguros para el tratamiento de residuos. La alternativa estudiada puede llegar a permitir la reducción de la cantidad de arcilla requerida. En China, por ejemplo, existe una prohibición para el uso de bloques de arcilla debido a la gran cantidad que se consume y la escasez de dicho material en este país [1]. Se ha estudiado el uso de diferentes residuos como material de reemplazo en bloques y estructuras de concreto y cemento sin resultados concluyentes, dadas las dificultades en el proceso de fraguado y el deterioro de la calidad del producto final [24]. La disposición final de residuos sólidos es un problema ambiental crítico en la mayoría de paí- ses latinoamericanos y solamente algunas de las principales ciudades cuentan con métodos de disposición adecuados [5]. El tratamiento y disposición, ambientalmente seguros y económicamente viables, de los lodos provenientes de los sistemas de alcantarillado, del tratamiento de las aguas residuales domésticas y de las plantas de tratamiento de aguas residuales industriales, constituye un reto de ingeniería para nuestros países.

\section{MARCO CONCEPTUAL}

Souza Santos [6], define una arcilla, en forma general, como un material terroso, de baja granulometría y que presenta plasticidad cuando es mezclado con cierta cantidad de agua. Las arcillas están constituidas por partículas cristalinas extremadamente pequeñas $(<2 \mu \mathrm{m})$ denominadas "minerales de arcilla", de naturaleza inorgánica, compuesta principalmente por $\mathrm{Si}, \mathrm{Al}, \mathrm{H}_{2} \mathrm{O}$ $\mathrm{y}$, frecuentemente, cantidades apreciables de $\mathrm{Fe}$ y metales alcalinos y alcalino- térreos. Además de minerales, una arcilla puede contener materia orgánica y otros compuestos no cristalinos o amorfos.

La arcilla es el material más utilizado para la fabricación de ladrillos y piezas de mampostería en Colombia. Dentro de la mampostería se conocen tres tipos básicos de unidades de arcilla cocida, que se distinguen según la disposición de sus 
perforaciones y del volumen que éstas ocupen. De acuerdo con esto, las unidades pueden ser de perforación vertical (PV); perforación horizontal $(\mathrm{PH})$ y macizos $(\mathrm{M})$. La aplicación de cada tipo de unidad y las respectivas características físicas deben estar de acuerdo con los cálculos y requisitos establecidos en la Reglamento de Sismoresistencia NSR-10, con el fin de que se garantice la estabilidad de la estructura [7]. Las propiedades que deben cumplir las unidades de mampostería son:

- Absorción de agua: es la cantidad de agua que penetra en los poros de la unidad con relación al peso seco.

- Resistencia mecánica a la compresión: es la mínima resistencia nominal de la mampostería a compresión, medida sobre el área transversal neta y sobre la cual se basa su diseño.

- Tasa inicial de absorción: es la medida de la cantidad de agua que absorbe una unidad de mampostería de arcilla en contacto con el agua por unidad de área, durante un minuto.

Las propiedades descritas anteriormente pueden tener requisitos adicionales y características especiales de diseño y calidad, por razones de exigencias acústicas, térmicas, de resistencia al fuego, arquitectónicas o constructivas, pero deben mantener los requisitos de absorción de agua y resistencia a la compresión para su uso principal -interior, exterior o estructural-. Las unidades de mampostería arquitectónica pueden tener formas, texturas y acabados libres, pero, igualmente, deben mantener los requisitos pertinentes para su aplicación principal.

\section{MATERIALES Y MÉTODOS}

A continuación se describen los ensayos de absorción de agua y resistencia mecánica a la compresión realizados a los ladrillos de prueba, así como el diseño experimental utilizado:
Absorción de agua: se siguió el procedimiento descrito en la Norma Técnica Colombiana NTC 4017 [8], que equivale al procedimiento de la American Society for Testing and Materials ASTM C67. Corresponde al volumen de agua recogida por una unidad, durante la inmersión a las condiciones prescritas en relación con el peso seco inicial de la misma. El resultado de la absorción es la diferencia entre la absorción fría y la caliente. Dicha relación no puede ser inferior a 0,8 .

Resistencia mecánica a la compresión: las unidades de mampostería de arcilla cocida deben cumplir con la resistencia mínima a la compresión, de acuerdo con el procedimiento de la NTC 4017. Para los ladrillos de perforación vertical la resistencia neta se calcula dividiendo la carga de rotura o de falla, por el área neta de la sección perpendicular a la carga -se descuentan las áreas de celdas y perforaciones- y en los ladrillos macizos, la resistencia neta y la resistencia bruta son iguales por que se calculan dividiendo por el área de apoyo de los ladrillos.

\subsection{Diseño experimental}

Para encontrar la proporción óptima de lodo en la mezcla con arcilla, se aplicó un diseño de experimentos de efectos fijos unifactorial, en razón a que el objeto del estudio consistía en encontrar la mejor respuesta del lodo frente a la resistencia mecánica a la compresión. El desarrollo de las pruebas se hizo de tal manera que se controlaron las demás variables que pudieran afectar esta variable. La ecuación (1) es el modelo matemático asociado utilizando la herramienta SAS

$$
Y i j k=\mu+A C i+\varepsilon i j+\eta i j k
$$

Con:

$i=0,1,2,3,4$ y 5 .

$j=1,2$ y 3 .

$k=1,2,3,4$ y 5 
Donde:

Yijk $=$ Variable respuesta, resistencia mecánica medida en Mega Pascales.

$\mu \quad=\quad$ Valor medio total de la resistencia mecánica medido en $\mathrm{M} \mathrm{Pa}$.

$A C i=$ Nivel de concentración de lodo agregado a la arcilla.

sij $=$ Error experimental debido a las unidades experimentales.

$\eta i j k=$ Error de muestreo debido a las unidades muestrales.

Se planteó como hipótesis que la concentración de lodo en la arcilla afecta la resistencia a la compresión de los ladrillos, mientras que la hipótesis nula para este diseño experimental fue que la concentración de lodos en la arcilla no la afecta.

\section{RESULTADOS Y DISCUSIÓN}

La caracterización de la arcilla y los lodos provenientes de la PTAR de la empresa GM COLMOTORES, a partir de DRX y FRX (tabla 1),
Tabla 1. Composición de lodos y arcilla

\begin{tabular}{|l|c|l|c|}
\hline \multicolumn{2}{|c|}{ LODOS PTAR } & \multicolumn{2}{c|}{ ARCILLA } \\
\hline Sustancia & $\%$ & Sustancia & $\%$ \\
\hline $\mathrm{SiO} 2$ & 41,0 & $\mathrm{SiO} 2$ & 58,5 \\
\hline $\mathrm{Al} 2 \mathrm{O} 3$ & 16,0 & $\mathrm{Al} 2 \mathrm{O} 3$ & 24,7 \\
\hline $\mathrm{CaO}$ & 12,5 & $\mathrm{CaO}$ & 0,5 \\
\hline $\mathrm{MgO}$ & 2,5 & $\mathrm{MgO}$ & 0,3 \\
\hline Fe2O3 & 8,6 & $\mathrm{Fe} 2 \mathrm{O} 3$ & 4,8 \\
\hline $\mathrm{P} 2 \mathrm{O} 5$ & 8,4 & $\mathrm{P} 2 \mathrm{O} 5$ & 0,5 \\
\hline $\mathrm{N} 2 \mathrm{O} 5$ & 3,5 & $\mathrm{TiO} 2$ & 0,9 \\
\hline $\mathrm{CaCO} 3$ & 2,5 & $\mathrm{~K} 2 \mathrm{O}$ & 1,5 \\
\hline K2O & 0,8 & MnO & 0,8 \\
\hline MnO & 0,7 & Humedad & 7,5 \\
\hline TiO2 & 1,1 & & \\
\hline Fe2SiO4 & 0,4 & & \\
\hline Humedad & 2,0 & & \\
\hline
\end{tabular}

Fuente: elaboración propia

confirma una alta afinidad entre ellos. Los lodos utilizados presentan un alto contenido tanto de materia inorgánica como de minerales de sílice junto con óxidos de diferentes metales, propios del proceso.

La figura 1 muestra los difractogramas obtenidos en el análisis de Difracción de Rayos X de

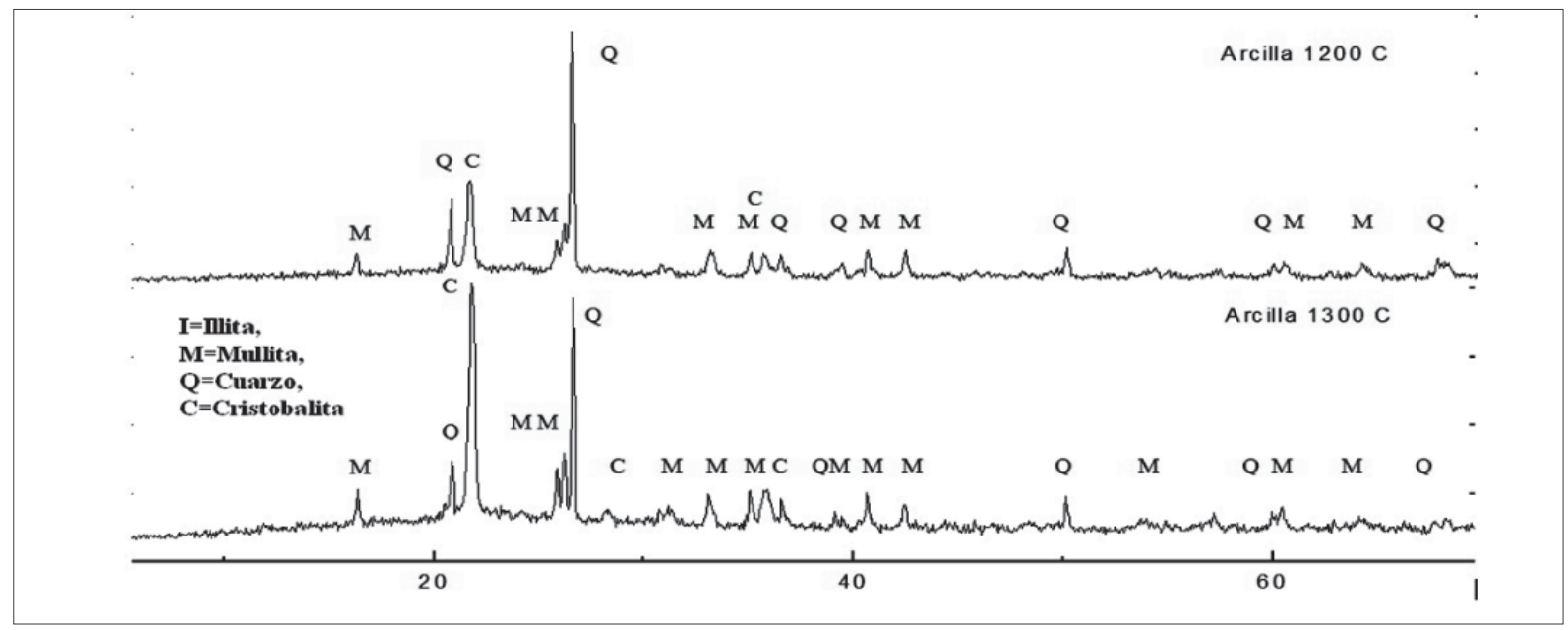

Figura 1. Difractogramas para la arcilla en (número de conteo $Y$ vs. Angulo $X$ )

Fuente: elaboración propia 


\section{investigación}

Tabla 2. Ensayo de resistencia a la compresión y absorción de agua para bloques No. 5 fabricados con mezcla de arcilla y lodo (Proporción 100:0 respectivamente)

\begin{tabular}{|c|c|c|c|c|c|}
\hline \multirow{2}{*}{$\begin{array}{c}\text { Muestra } \\
\text { No. }\end{array}$} & \multirow{2}{*}{$\begin{array}{l}\text { Área } \\
\mathrm{cm}^{2}\end{array}$} & \multirow{2}{*}{$\begin{array}{l}\text { Carga de } \\
\text { rotura }(\mathrm{kg})\end{array}$} & \multicolumn{2}{|c|}{ Resistencia } & \multirow{2}{*}{$\begin{array}{c}\text { Absorción } \\
\text { de agua } \%^{2}\end{array}$} \\
\hline & & & $\mathrm{kg} / \mathrm{cm}^{2}$ & $\mathrm{MPa}^{1}$ & \\
\hline \multicolumn{6}{|c|}{ Prueba 1 - Nov 04} \\
\hline 1 & 359 & 13500 & 37,60 & 3,69 & 11,70 \\
\hline 2 & 356 & 15500 & 43,54 & 4,27 & 12,30 \\
\hline 3 & 360 & 14600 & 40,56 & 3,98 & 13,20 \\
\hline 4 & 357 & 13900 & 38,94 & 3,82 & 12,20 \\
\hline 5 & 358 & 14000 & 39,11 & 3,83 & 12,80 \\
\hline Promedio & 358 & 14300 & 39,95 & 3,92 & 12,44 \\
\hline \multicolumn{6}{|c|}{ Prueba 2-feb. 05} \\
\hline 1 & 357 & 12800 & 35,85 & 3,52 & 12,20 \\
\hline 2 & 356 & 14700 & 41,29 & 4,05 & 13,00 \\
\hline 3 & 357 & 13900 & 38,94 & 3,82 & 12,50 \\
\hline 4 & 360 & 14600 & 40,56 & 3,98 & 13,30 \\
\hline 5 & 356 & 12600 & 35,39 & 3,47 & 12,20 \\
\hline Promedio & 357 & 13720 & 38,41 & 3,77 & 12,64 \\
\hline \multicolumn{6}{|c|}{ Prueba 3-jun. 05} \\
\hline 1 & 358 & 12500 & 34,92 & 3,42 & 12,20 \\
\hline 2 & 356 & 14900 & 41,85 & 4,10 & 11,90 \\
\hline 3 & 360 & 11600 & 32,22 & 3,16 & 13,10 \\
\hline 4 & 357 & 15900 & 44,54 & 4,37 & 12,10 \\
\hline 5 & 360 & 11900 & 33,06 & 3,24 & 12,20 \\
\hline Promedio & 358 & 13360 & 37,32 & 3,66 & 12,30 \\
\hline \multicolumn{6}{|c|}{$\begin{aligned} 1 \text { Valor mínimo según NTC } 4205=>3 \\
2 \text { Valor máximo según NTC } 4205=<14\end{aligned}$} \\
\hline
\end{tabular}

Fuente: elaboración propia

las arcillas utilizadas a $1200{ }^{\circ} \mathrm{C}$ y $1300^{\circ} \mathrm{C}$. Como resultado de este análisis, se aprecia que estas arcillas, compuestas principalmente por caolinita (M), illita (I), montmorillonita (M) y cuarzo (Q), con la perdida de humedad y el reacomodamiento de su estructura, se forman en minerales muy estables conocidos como mullita y cristobalita (C) además del cuarzo $(\mathrm{Q})$, que según diferentes autores, confieren las propiedades mecánicas de los ladrillos [9]. 


\section{investigación}

Tabla 3. Ensayo de resistencia a la compresión y absorción de agua para bloques No. 5 fabricados con mezcla de arcilla y lodo (Proporción 99:1 respectivamente).

\begin{tabular}{|c|c|c|c|c|c|}
\hline \multirow{2}{*}{$\begin{array}{c}\text { Muestra } \\
\text { No. }\end{array}$} & \multirow{2}{*}{$\begin{array}{c}\text { Área } \\
\mathrm{cm}^{2}\end{array}$} & \multirow{2}{*}{$\begin{array}{l}\text { Carga de } \\
\text { rotura }(\mathrm{kg})\end{array}$} & \multicolumn{2}{|c|}{ Resistencia } & \multirow{2}{*}{$\begin{array}{l}\text { Absorción } \\
\text { de agua \%(2) }\end{array}$} \\
\hline & & & $\mathrm{kg} / \mathrm{cm}^{2}$ & $\mathrm{M} \mathrm{Pa}^{(1)}$ & \\
\hline \multicolumn{6}{|c|}{ Prueba 1 - Nov 04} \\
\hline 1 & 359 & 14500 & 40,39 & 3,96 & 12,50 \\
\hline 2 & 358 & 13600 & 37,99 & 3,72 & 12,10 \\
\hline 3 & 356 & 14800 & 41,57 & 4,08 & 12,90 \\
\hline 4 & 356 & 14100 & 39,61 & 3,88 & 13,30 \\
\hline 5 & 360 & 13900 & 38,61 & 3,79 & 12,90 \\
\hline Promedio & 357,8 & 14180 & 39,63 & 3,89 & 12,74 \\
\hline \multicolumn{6}{|c|}{ Prueba 2-feb. 05} \\
\hline 1 & 356 & 13700 & 38,48 & 3,77 & 12,70 \\
\hline 2 & 358 & 14500 & 40,50 & 3,97 & 12,40 \\
\hline 3 & 359 & 13200 & 36,77 & 3,60 & 13,20 \\
\hline 4 & 357 & 14600 & 40,90 & 4,01 & 13,40 \\
\hline 5 & 356 & 13900 & 39,04 & 3,83 & 12,40 \\
\hline Promedio & 357 & 13980 & 39,14 & 3,84 & 12,82 \\
\hline \multicolumn{6}{|c|}{ Prueba 3-jun. 05} \\
\hline 1 & 357 & 14600 & 40,90 & 4,01 & 13,50 \\
\hline 2 & 359 & 14800 & 41,23 & 4,04 & 12,30 \\
\hline 3 & 360 & 13900 & 38,61 & 3,79 & 13,10 \\
\hline 4 & 359 & 13500 & 37,60 & 3,69 & 12,50 \\
\hline 5 & 357 & 14100 & 39,50 & 3,87 & 13,20 \\
\hline Promedio & 358 & 14180 & 39,57 & 3,88 & 12,92 \\
\hline \multicolumn{6}{|c|}{$\begin{array}{l}\text { (1) Valor mínimo según NTC } 4205=>3 \\
\text { (2) Valor máximo según NTC } 4205=<14\end{array}$} \\
\hline
\end{tabular}

Fuente: elaboración propia

Lo anterior evidencia que, aparentemente, el material adquiere una gran estabilidad con la temperatura, lo cual quiere decir que no se va a descomponer y tampoco va a desprender, producir o generar ningún tipo de sustancia al someterlo a condiciones elevadas de temperatura. Esta propiedad es bastante apreciable dado el uso que se pretende dar a los ladrillos. 


\section{investigación}

Tabla 4. Ensayo de resistencia a la compresión y absorción de agua para bloques No. 5 fabricados con mezcla de arcilla y lodo (Proporción 95:5 respectivamente)

\begin{tabular}{|c|c|c|c|c|c|}
\hline \multirow{2}{*}{$\begin{array}{l}\text { Muestra } \\
\text { No. }\end{array}$} & \multirow{2}{*}{$\begin{array}{c}\text { Área } \\
\mathrm{cm}^{2}\end{array}$} & \multirow{2}{*}{$\begin{array}{l}\text { Carga de } \\
\text { rotura }(\mathbf{k g})\end{array}$} & \multicolumn{2}{|c|}{ Resistencia } & \multirow{2}{*}{$\begin{array}{l}\text { Absorción } \\
\text { de agua } \%{ }^{(2)}\end{array}$} \\
\hline & & & $\mathrm{kg} / \mathrm{cm}^{2}$ & $\mathbf{M P a} a^{(1)}$ & \\
\hline \multicolumn{6}{|c|}{ Prueba 1 - nov. 04} \\
\hline 1 & 357 & 15100 & 42,30 & 4,10 & 12,90 \\
\hline 2 & 358 & 16000 & 44,69 & 4,40 & 12,60 \\
\hline 3 & 358 & 18200 & 50,84 & 5,00 & 12,90 \\
\hline 4 & 356 & 14600 & 41,01 & 4,00 & 13,50 \\
\hline 5 & 360 & 17400 & 48,33 & 4,70 & 13,40 \\
\hline Promedio & 358 & 16260 & 45,43 & 4,44 & 13,06 \\
\hline \multicolumn{6}{|c|}{ Prueba 2 - feb. 05} \\
\hline 1 & 360 & 17300 & 48,06 & 4,70 & 12,90 \\
\hline 2 & 360 & 15300 & 42,50 & 4,20 & 12,70 \\
\hline 3 & 356 & 19300 & 54,21 & 5,30 & 13,10 \\
\hline 4 & 358 & 15200 & 42,46 & 4,20 & 12,50 \\
\hline 5 & 357 & 16100 & 45,10 & 4,40 & 13,50 \\
\hline Promedio & 358 & 16640 & 46,47 & 4,56 & 12,94 \\
\hline \multicolumn{6}{|c|}{ Prueba 3 - jun. 05} \\
\hline 1 & 358 & 18200 & 50,84 & 5,00 & 13,20 \\
\hline 2 & 356 & 15300 & 42,98 & 4,20 & 12,50 \\
\hline 3 & 359 & 13800 & 38,44 & 3,80 & 13,30 \\
\hline 4 & 357 & 16700 & 46,78 & 4,60 & 12,90 \\
\hline 5 & 357 & 18500 & 51,82 & 5,10 & 12,70 \\
\hline Promedio & 357 & 16500 & 46,17 & 4,54 & 12,92 \\
\hline $\begin{array}{l}\text { (1) Valor mínim } \\
\text { (2) Valor máxin }\end{array}$ & $\begin{array}{l}4205 \\
4205\end{array}$ & & & & \\
\hline
\end{tabular}

Fuente: elaboración propia

\subsection{Análisis de calidad de los ladrillos}

Los resultados de las pruebas de absorción en frío, carga rotura y resistencia, para los ladrillos elaborados con las diferentes mezclas de arcilla-lodo, de acuerdo con los procedimientos de la NTC 4205 [10] se presentan en las tablas 2 a 7.
De los resultados obtenidos, se observa que la resistencia a la compresión de los ladrillos fabricados con todas las proporciones, excepto 60:40, se encuentra dentro de los estándares establecidos para el país. Se observa un ligero aumento de la resistencia a medida que se aumenta la concentración de lodos, lo cual es favorable. Sin embargo, 


\section{investigación}

Tabla 5. Ensayo de resistencia a la compresión y absorción de agua para bloques No. 5 fabricados con mezcla de arcilla y lodo (Proporción 90:10 respectivamente)

\begin{tabular}{|c|c|c|c|c|c|}
\hline \multirow{2}{*}{$\begin{array}{c}\text { Muestra } \\
\text { No. }\end{array}$} & \multirow{2}{*}{$\begin{array}{l}\text { Área } \\
\mathrm{cm}^{2}\end{array}$} & \multirow{2}{*}{$\begin{array}{l}\text { Carga de } \\
\text { rotura }(\mathbf{k g})\end{array}$} & \multicolumn{2}{|c|}{ Resistencia } & \multirow{2}{*}{$\begin{array}{l}\text { Absorción } \\
\text { de agua \% }{ }^{(2)}\end{array}$} \\
\hline & & & $\mathrm{kg} / \mathrm{cm}^{2}$ & $\mathbf{M P a} \mathbf{P}^{(1)}$ & \\
\hline \multicolumn{6}{|c|}{ Prueba 1 - nov. 04} \\
\hline 1 & 358 & 15700 & 43,85 & 4,30 & 12,90 \\
\hline 2 & 358 & 16500 & 46,09 & 4,52 & 13,40 \\
\hline 3 & 356 & 17500 & 49,16 & 4,82 & 13,50 \\
\hline 4 & 360 & 14900 & 41,39 & 4,06 & 13,30 \\
\hline 5 & 357 & 17800 & 49,86 & 4,89 & 13,00 \\
\hline Promedio & 358 & 16480 & 46,07 & 4,52 & 13,22 \\
\hline \multicolumn{6}{|c|}{ Prueba 2 - feb. 05} \\
\hline 1 & 356 & 17600 & 49,44 & 4,85 & 13,50 \\
\hline 2 & 359 & 15900 & 44,29 & 4,34 & 13,10 \\
\hline 3 & 360 & 19600 & 54,44 & 5,34 & 13,40 \\
\hline 4 & 357 & 15500 & 43,42 & 4,26 & 12,80 \\
\hline 5 & 360 & 16500 & 45,83 & 4,49 & 13,20 \\
\hline Promedio & 358 & 17020 & 47,48 & 4,66 & 13,20 \\
\hline \multicolumn{6}{|c|}{ Prueba 3 - jun. 05} \\
\hline 1 & 360 & 18600 & 51,67 & 5,07 & 13,40 \\
\hline 2 & 358 & 16000 & 44,69 & 4,38 & 13,30 \\
\hline 3 & 356 & 14500 & 40,73 & 3,99 & 13,20 \\
\hline 4 & 357 & 16800 & 47,06 & 4,61 & 13,50 \\
\hline 5 & 358 & 18900 & 52,79 & 5,18 & 13,10 \\
\hline Promedio & 358 & 16960 & 47,39 & 4,65 & 13,30 \\
\hline $\begin{array}{l}\text { (1) Valor mínim } \\
\text { (2) Valor máxim }\end{array}$ & 4205 & & & & \\
\hline
\end{tabular}

Fuente: elaboración propia

la absorción de agua también aumenta a medida que aumenta la concentración de lodo en los bloques, lo que es altamente desfavorable, porque una mayor absorción de agua reduce la estabilidad de la pieza en el tiempo.

Al examinar los diagramas de caja, para cada nivel de concentración de lodos, se observa que la resistencia mecánica a la compresión se incrementa, cuando la concentración de lodo aumenta, hasta cerca del $10 \%$ y que por encima de éste se aprecia un ligero descenso. Con base en este análisis de la figura 2, se infiere que la concentración de lodo en la arcilla sí afecta la resistencia mecánica de los ladrillos y que la concentración de lodo que produce la máxima resistencia a la com- 


\section{investigación}

Tabla 6. Ensayo de resistencia a la compresión y absorción de agua para bloques No. 5 fabricados con mezcla de arcilla y lodo (Proporción 90:10 respectivamente)

\begin{tabular}{|c|c|c|c|c|c|}
\hline \multirow{2}{*}{$\begin{array}{c}\text { Muestra } \\
\text { No. }\end{array}$} & \multirow{2}{*}{$\begin{array}{c}\text { Área } \\
\mathrm{cm}^{2}\end{array}$} & \multirow{2}{*}{$\begin{array}{l}\text { Carga de } \\
\text { rotura }(\mathrm{kg})\end{array}$} & \multicolumn{2}{|c|}{ Resistencia } & \multirow{2}{*}{$\begin{array}{c}\text { Absorción } \\
\text { de agua } \% \text { (2) }\end{array}$} \\
\hline & & & $\mathrm{kg} / \mathrm{cm}^{2}$ & $\mathrm{MPa}^{(1)}$ & \\
\hline \multicolumn{6}{|c|}{ Prueba 1 - nov. 04} \\
\hline 1 & 358 & 15700 & 43,85 & 4,30 & 12,90 \\
\hline 2 & 358 & 16500 & 46,09 & 4,52 & 13,40 \\
\hline 3 & 356 & 17500 & 49,16 & 4,82 & 13,50 \\
\hline 4 & 360 & 14900 & 41,39 & 4,06 & 13,30 \\
\hline 5 & 357 & 17800 & 49,86 & 4,89 & 13,00 \\
\hline Promedio & 358 & 16480 & 46,07 & 4,52 & 13,22 \\
\hline \multicolumn{6}{|c|}{ Prueba 2 - feb. 05} \\
\hline 1 & 356 & 17600 & 49,44 & 4,85 & 13,50 \\
\hline 2 & 359 & 15900 & 44,29 & 4,34 & 13,10 \\
\hline 3 & 360 & 19600 & 54,44 & 5,34 & 13,40 \\
\hline 4 & 357 & 15500 & 43,42 & 4,26 & 12,80 \\
\hline 5 & 360 & 16500 & 45,83 & 4,49 & 13,20 \\
\hline Promedio & 358 & 17020 & 47,48 & 4,66 & 13,20 \\
\hline \multicolumn{6}{|c|}{ Prueba 3 - jun. 05} \\
\hline 1 & 360 & 18600 & 51,67 & 5,07 & 13,40 \\
\hline 2 & 358 & 16000 & 44,69 & 4,38 & 13,30 \\
\hline 3 & 356 & 14500 & 40,73 & 3,99 & 13,20 \\
\hline 4 & 357 & 16800 & 47,06 & 4,61 & 13,50 \\
\hline 5 & 358 & 18900 & 52,79 & 5,18 & 13,10 \\
\hline Promedio & 358 & 16960 & 47,39 & 4,65 & 13,30 \\
\hline $\begin{array}{l}\text { (1) Valor mínim } \\
\text { (2) Valor máxim }\end{array}$ & $\begin{array}{l}4205 \\
4205\end{array}$ & & & & \\
\hline
\end{tabular}

Fuente: elaboración propia

presión está entre el $5 \%$ y el $10 \%$. Así mismo, se deduce que una concentración de $40 \%$ de lodo en la mezcla, genera piezas que no cumplen con las normas de calidad.

Se corrió el procedimiento estadístico General Linear Model GLM con la herramienta Statistical
Analysis System SAS (tabla 8), para contrastar el siguiente sistema de hipótesis:

Ho: $\mu 0=\mu 1=\mu 2=\mu 3=\mu 4=\mu 5$

Ha: Al menos un par de medias $\mu \mathrm{i} \neq \mu \mathrm{j}$ para $\mathrm{i} \neq \mathrm{j}$ 


\section{investigación}

Tabla 7. Ensayo de resistencia a la compresión y absorción de agua para bloques No. 5 fabricados con mezcla de arcilla y lodo (Proporción 80:20 respectivamente)

\begin{tabular}{|c|c|c|c|c|c|}
\hline \multirow{2}{*}{$\begin{array}{c}\text { Muestra } \\
\text { No. }\end{array}$} & \multirow{2}{*}{$\begin{array}{l}\text { Área } \\
\mathrm{cm}^{2}\end{array}$} & \multirow{2}{*}{$\begin{array}{l}\text { Carga de } \\
\text { rotura }(\mathrm{kg})\end{array}$} & \multicolumn{2}{|c|}{ Resistencia } & \multirow{2}{*}{$\begin{array}{l}\text { Absorción } \\
\text { de agua } \%(2)\end{array}$} \\
\hline & & & $\mathrm{kg} / \mathrm{cm}^{2}$ & $\mathrm{MPa}^{(1)}$ & \\
\hline \multicolumn{6}{|c|}{ Prueba 1 - nov. 04} \\
\hline 1 & 356 & 12500 & 35,11 & 3,44 & 14,00 \\
\hline 2 & 358 & 12600 & 35,20 & 3,45 & 13,70 \\
\hline 3 & 359 & 11900 & 33,15 & 3,25 & 13,00 \\
\hline 4 & 360 & 11600 & 32,22 & 3,16 & 13,20 \\
\hline 5 & 359 & 12200 & 33,98 & 3,33 & 13,60 \\
\hline Promedio & 358 & 12160 & 33,93 & 3,33 & 13,50 \\
\hline \multicolumn{6}{|c|}{ Prueba 2 - feb. 05} \\
\hline 1 & 359 & 12400 & 34,54 & 3,39 & 13,70 \\
\hline 2 & 359 & 11800 & 32,87 & 3,22 & 13,90 \\
\hline 3 & 360 & 12700 & 35,28 & 3,46 & 13,50 \\
\hline 4 & 357 & 11500 & 32,21 & 3,16 & 14,10 \\
\hline 5 & 356 & 12300 & 34,55 & 3,39 & 13,20 \\
\hline Promedio & 358 & 12140 & 33,89 & 3,32 & 13,68 \\
\hline \multicolumn{6}{|c|}{ Prueba 3 - jun. 05} \\
\hline 1 & 357 & 12800 & 35,85 & 3,52 & 13,20 \\
\hline 2 & 359 & 12600 & 35,10 & 3,44 & 13,70 \\
\hline 3 & 358 & 11800 & 32,96 & 3,23 & 14,20 \\
\hline 4 & 360 & 12300 & 34,17 & 3,35 & 13,50 \\
\hline 5 & 356 & 11700 & 32,87 & 3,22 & 13,10 \\
\hline Promedio & 358 & 12240 & 34,19 & 3,35 & 13,54 \\
\hline
\end{tabular}

(1) Valor mínimo según NTC $4205=>3$

(2) Valor máximo según NTC $4205=<14$

Fuente: elaboración propia

Según esta información, se observa que el F-valor $<, 0001$ correspondiente a la concentración de lodos, rechaza la hipótesis nula de igualdad entre la resistencias mecánica a la compresión para los seis niveles de concentración analizados. Además, el F-valor correspondiente al Error Experimental 0,9964 $>0,05$ no rechaza Ho: $\sigma 2 \mathrm{e}=0$, $1 \mathrm{o}$ cual sugiere que no es importante hacer submuestreo en este experimento.

Como se rechazó la hipótesis de igualdad de medias, se requiere contrastar las siguientes hipótesis: 


\section{investigación}

Tabla 8. Ensayo de resistencia a la compresión y absorción de agua para bloques No. 5 fabricados con mezcla de arcilla y lodo (Proporción 60:40 respectivamente)

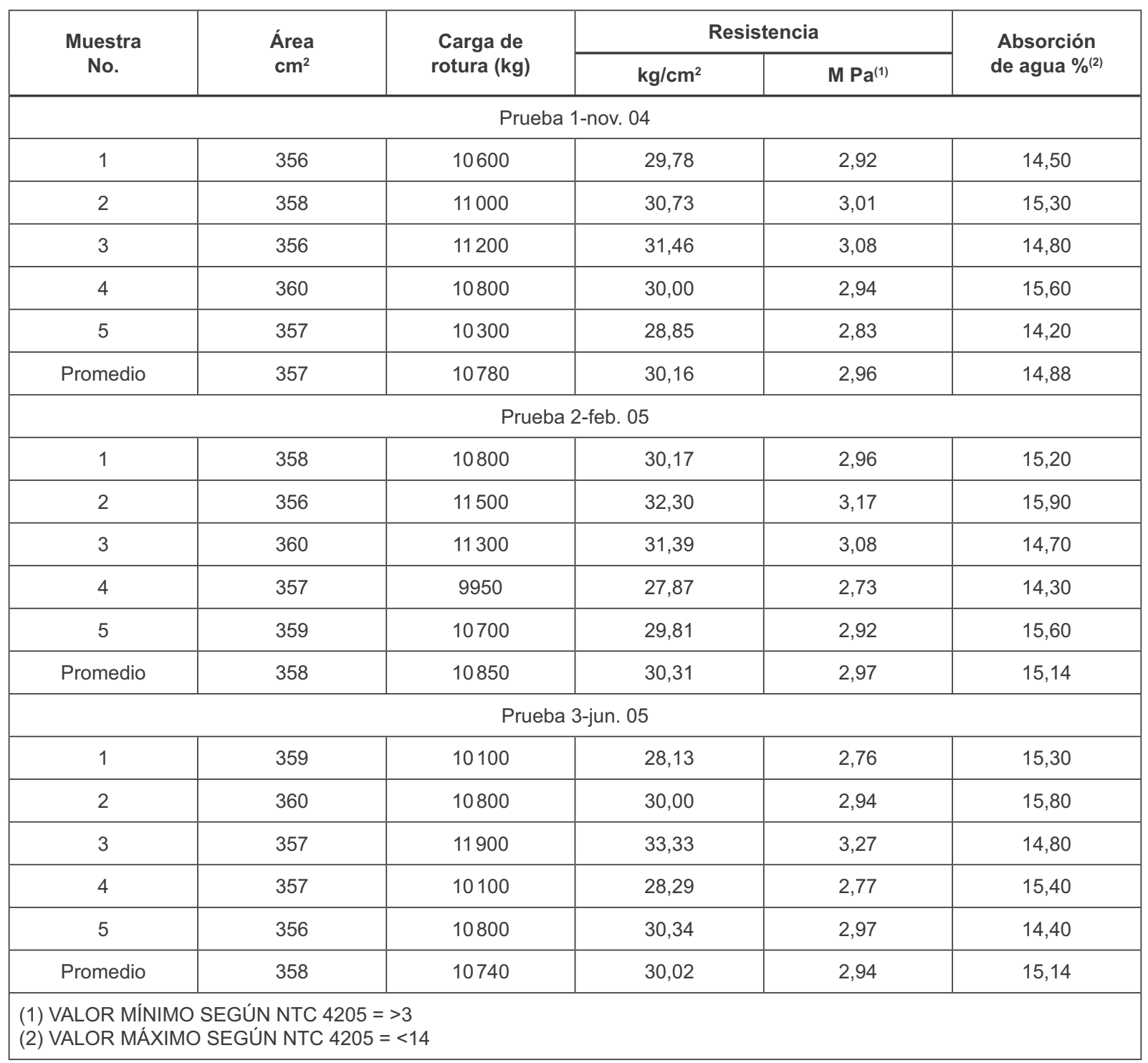

Fuente: elaboración propia

Ho: $\mu 2=\mu 3$

Ha: $\mu 2 \neq \mu 3$

Como el P-valor $=0,553$, la prueba t no rechaza la hipótesis nula de igualdad de medias entre los tratamientos 2 y 3 ; es decir, no existe diferencia significativa entre las medias de las concentraciones del $5 \%$ y $10 \%$ de lodo. También se quiere determinar si existe diferencia o no entre la resistencia mecánica a la compresión entre el tratamiento de $0 \%$ de lodos y el de $10 \%$ de lodos.

Ho: $\mu 0=\mu 3$

Ha: $\mu 0 \neq \mu 3$ 


\section{investigación}

Tabla 9. Resultados análisis estadístico de hipótesis - Procedimiento GLM

\begin{tabular}{|c|c|c|c|c|c|}
\hline \multicolumn{2}{|c|}{ Variable dependiente: compresión } & & & \\
\hline Fuente & DF & Suma de Cuadrados & Cuadrado de la media & F-valor & Pr $>$ F \\
\hline Modelo & 17 & 31,50696556 & 1,85335092 & 17,97 & $<, 0001$ \\
\hline Error & 72 & 7,42520000 & 0,10312778 & & \\
\hline Total & 89 & 38,93216556 & & & \\
\hline R-cuadrado & Coef Var & Raiz MSE & Compresion Media & & \\
\hline 0,809279 & 8,355883 & 0,321135 & 3.843 .222 & & Pr $>$ F \\
\hline Fuente & DF & Tipo I SS & Cuadrado de la media & F-valor & $<, 0001$ \\
\hline AC & 5 & 31,22394 & 6,2447878 & 60,55 & 0,9964 \\
\hline REPLICA(AL) & 12 & 0,28302667 & 0,02358556 & 0,23 & 0 \\
\hline
\end{tabular}

Fuente: elaboración propia

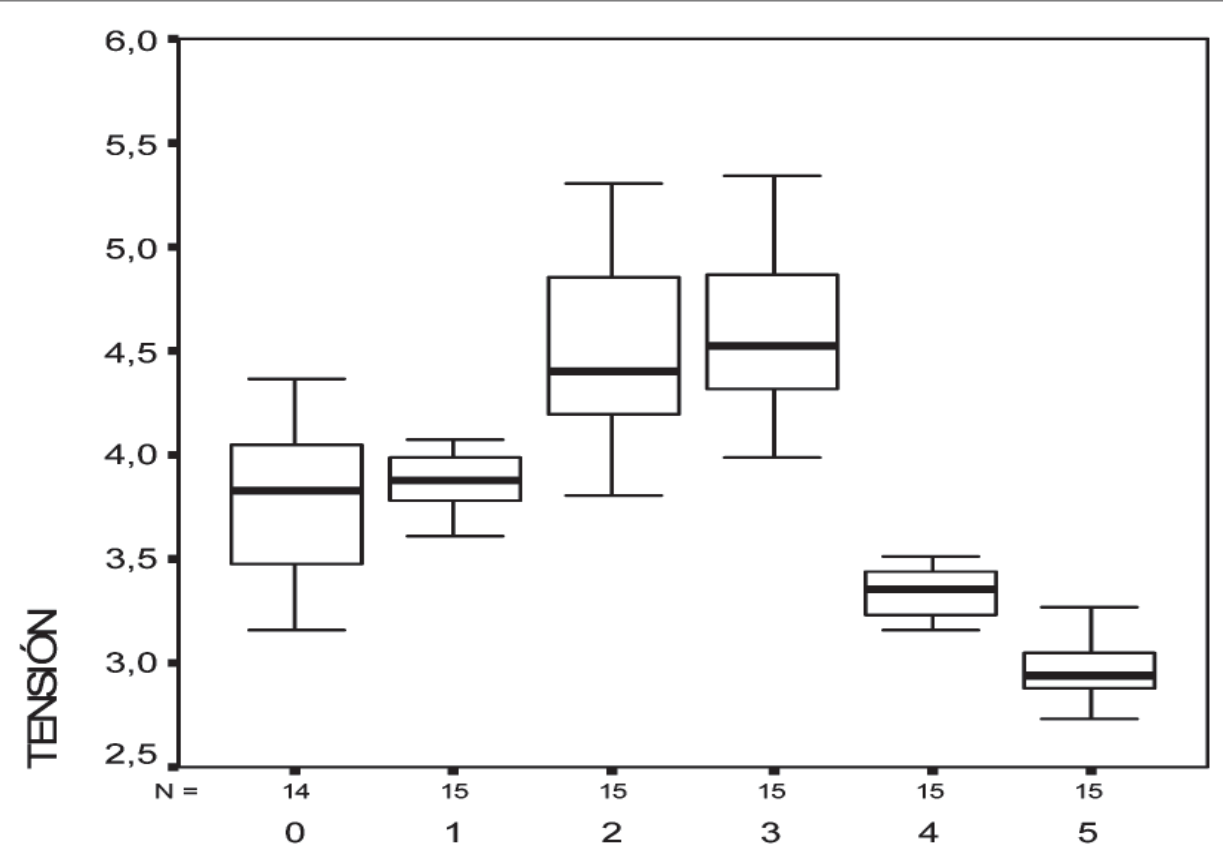

$\mathrm{AC}$

Figura 2. Porcentaje de concentración de lodo vs resistencia mecánica de los ladrillos

Fuente: elaboración propia 
Para esta prueba, con un P-Valor $=0,000$ se rechaza la hipótesis de igualdad de medias, luego, la diferencia media entre los tratamientos cero y tres es estadísticamente significativa.

Con base en el tratamiento estadístico, se puede inferir que las mejores respuestas se dan con el tratamiento 3, que corresponde a la concentración de lodos en la arcilla de $10 \%$. Esta proporción permite obtener la resistencia máxima a la compresión y utiliza la mayor cantidad posible de lodos sin afectar drásticamente la cantidad máxima de absorción de agua permitida.

\section{CONCLUSIONES}

Se concluye que la adición de lodos, en fracciones menores a $10 \%$ en peso a la arcilla utilizada en el proceso ladrillero, no reduce la resistencia mecánica de los ladrillos fabricados en estas condiciones. En los porcentajes de mezcla $95-5$ y $90-$ 10 , se presenta un ligero aumento en la resistencia a la compresión, mientras que en la proporción 80-20 este aumento es menor, y en la proporción 60-40 la resistencia se reduce significativamente incluso por debajo del nivel mínimo establecido por la norma.

Los porcentajes de absorción de humedad se incrementan a medida que se aumenta la proporción de mezcla, llegando incluso a superar el valor permisible para el caso de la proporción 60-40.

De acuerdo con el análisis estadístico de los resultados obtenidos, el rango o el porcentaje de lodos más adecuado para hacer la mezcla con la arcilla para producir ladrillos, se encuentra entre el 5 y el $10 \%$.

Para la aplicación a escala real de esta alternativa se hace necesario llevar a cabo estudios detallados de caracterización y análisis elemental de los lodos y las arcillas para cada caso en particular, con el fin de conocer de manera precisa su composición y establecer así su grado de compatibilidad.

Los autores recomiendan complementar este estudio con otros similares, tanto en distintos lugares del país como con diferentes tipos de lodos industriales de aguas residuales y alcantarillados entre otros, para establecer de manera más precisa la viabilidad técnica, ambiental y económica de esta alternativa de tratamiento.

\section{FINANCIAMIENTO}

Para el desarrollo de este proyecto se contó con el apoyo económico de la empresa INAMCO y el auspicio de COLCIENCIAS.

\section{AGRADECIMIENTOS}

Los autores expresan su agradecimiento a las empresas INAMCO y Ladrillos Los Quiroga por el apoyo logístico y la colaboración prestada en el desarrollo de los ensayos a escala real sin lo cual no se habría podido desarrollar este proyecto. 


\section{REFERENCIAS}

[1] X. Lingling, G. Wei, W. Tao and Y. Nanru, "Study on fired bricks with replacing clay by fly ash in high volume ratio", Construction and Building Materials, Vol. 19, pp. 243-247, 2005.

[2] M. Allam, G. Garas and H. El Kady, "Recycled Chopped Rice Straw- Cement Bricks: Mechanical, Fire Resistance \& Economical Assessment", Australian Journal of Basic and Applied Sciences, Vol. 5, No. 2, pp. 27-33, 2011.

[3] G. Valdés, O. Reyes-Ortiz y G. González, "Aplicación de los residuos de hormigón en materiales de construcción", Ingeniería \& Desarrollo, Vol. 29, No. 1, pp. 17-33, 2011.

[4] C. Martínez, T. Cotes and F. Corpas, "Recovering wastes from the paper industry: Development of ceramic materials", Fuel processing technology, Vol. 103, pp. 117124, Nov. 2011.

[5] M. La Grega, P. Buckingham, and J. Evans, Hazardous Waste Management, Madrid: Ed McGraw-Hill Inc., 1994.
[6] P. Souza Santos, Tecnología de Argilas, Sao Paulo: Ed. Universidad de Sao Paulo, 1975.

[7] Reglamento colombiano de construcción sismoresistente, NCR-10, Ene. 2010.

[8] Métodos para muestreo y ensayos deunidades de mampostería y otrosproductos de arcilla, NTC 4017, Nov. 2005.

[9] C. García-Ubaque, Co-incineración de residuos sólidos con alto contenido de humedad en hornos ladrilleros de tipo continuo: Estudio de la estabilización de las cenizas en las arcillas utilizadas en el proceso, [Tesis $\mathrm{PhD}$ ], Universidad de los Andes, Colombia, 2007.

[10] Ingeniería Civil y Arquitectura. Unidades de mampostería de arcillacocida. Ladrillos y bloques cerámicos, NTC 4205, Oct. 2000. 